\title{
Flujos de mineralización en el embalse tropical Río Grande II (Antioquia, Colombia)
}

\author{
Yimmy Montoya M. ${ }^{1, *}$ \& John J. Ramírez R. ${ }^{2}$ \\ 1 Instituto de Biología, Universidad de Antioquia, apartado 1226, Medellín, Colombia. \\ 2 johnra77@yahoo.com. \\ * Autor responsable de la correspondencia: yimmymontoya3@ hotmail.com.
}

\section{RESUMEN}

Flujos de mineralización en la presa del embalse tropical Río Grande II (Antioquia, Colombia)

Se emplearon dos sistemas de cinco trampas de sedimentos colocados en la zona de la presa del embalse de Río grande II $\left(6^{\circ} 32,62^{\prime} \mathrm{N}, 75^{\circ} 27,27^{\prime} \mathrm{W}\right)$. Se tomaron muestras cada diez días entre abril de 2003 y julio del mismo año, con el fin de determinar cuál era la velocidad de la mineralización del material particulado y si ésta variaba en relación con los cambios en la altura de mezcla. Se presentó una tendencia en la que el amonio fluctuó con la altura de la capa de mezcla y con la temperatura del agua. Se evidenció que con el aumento en la altura de la capa de mezcla disminuía la mineralización del amonio y la profundidad a la que ocurre no variaba, manteniéndose la mayor descomposición de la materia orgánica sobre la segunda trampa. Para los nitratos, el consumo superó la liberación con el aumento de la temperatura del agua y de la altura de mezcla. El flujo de mineralización del fósforo presentó un valor negativo durante la investigación, lo que se asoció a una tasa de consumo permanente en el sistema. Además, el fósforo mostró una tendencia a disminuir su concentración con la profundidad, lo que no se ha reportado para un embalse tropical térmicamente estable.

Palabras clave: Mineralización, reciclaje, liberación, nutrientes, limnología tropical.

\begin{abstract}
Mineralization fluxes in the dam of the tropical Rio Grande II reservoir (Antioquia, Colombia)

Two systems of five sediment traps were placed in the area of the dam of the Rio Grande II reservoir $\left(6^{\circ} 32,62^{\prime} N, 75^{\circ} 27,27^{\prime}\right.$ W). Every ten days from April 2003 to July 2003 samples were taken with the purpose of determine the speed of mineralization of the particulate material and if it changes in relation to the mixing height. There was a tendency in which the ammonium fluctuated in relation to the height in the mixing layer and the temperature of the water. It was evidenced that with the increase in the height of the mixing layer the ammonium mineralization diminished; whereas the depth of mineralization didn't vary and the highest decomposition still occurred in the second trap. For nitrates, with the increase in temperature and the mixing height its consumption was higher than its liberation. Phosphorus mineralization flux showed a negative value during the investigation; it was associated to a rate of permanent consumption in the system. Phosphorus also showed a tendency to diminish its concentration with depth; this behavior has not been registered for a thermically stable tropical reservoir.
\end{abstract}

Keywords: Mineralization, recycling, release, nutrients, tropical limnology.

\section{INTRODUCCIÓN}

El término mineralización es el proceso que corresponde a la conversión de material orgánico partículado en componentes disueltos inorgánicos, tales como el amonio, el fósforo solu- ble reactivo, los nitratos y los nitritos. En el ambiente acuático la mineralización es realizada principalmente por bacterias y hongos, que convierten la materia orgánica partículada en minerales, agua y gas carbónico (Esteves, 1998). La bioreactividad de la materia orgánica disminuye 
con su fraccionamiento y su estado diagenético (Amon \& Benner, 1996). Axler et al. (1981) proponen evaluar funcionalmente la mineralización a través del amonio liberado.

El flujo de mineralización corresponde a la variación de la cantidad liberada o consumida del material inorgánico disuelto respecto a la profundidad. Los flujos de mineralización del material partículado dependen fuertemente de las especies de algas presentes, de la composición bioquímica, el tamaño molecular, la concentración de nutrientes inorgánicos, de la sedimentación, de la temperatura, del $\mathrm{pH}$, del potencial redox, de la hidrología y de las condiciones ambientales del reservorio (Cole \& Likens, 1979; Amon \& Benner, 1996; Perkins \& Underwood, 2001). Boers \& Hese (1988) encontraron que sólo temperaturas superiores a los $13{ }^{\circ} \mathrm{C}$ permiten la liberación de nutrientes, y Lehman (1980) planteó que dicha liberación depende del estado nutricional de las células algales. El reciclaje de nutrientes dentro de la columna de agua puede ocurrir debido a la muerte fisiológica del fitoplancton, a la autólisis ayudada por la acción bacteriana, al flujo de liberación por animales y a la mineralización. Rotíferos y Protozoos contribuyen al crecimiento bacteriano al liberar sustancias como enzimas líticas, de tipo proteasas extracelulares, que lisan productos de desecho de otros organismos y estimulan la actividad microbiana (Vadstein et al., 2003; Ratsak et al., 1996). Por ello, Håkanson \& Jansson (2002) plantean que una parte de los procesos de degradación externa que ocurren sobre los detritos son realizados por las bacterias y la autólisis (autogeneración de enzimas que realizan el proceso de descomposición de un organismo muerto), liberando un gran número de sustancias como las de tipo húmico. Alvarez et al., (1993) consideran que la fracción que se sedimenta varía inversamente con la intensidad de la descomposición y con la distancia de sedimentación (profundidad de atrapamiento). Una vez sedimentado el material sestónico, las algas bentónicas sirven como un enlace que transporta nitrógeno desde los sedimentos hasta la columna de agua. Las algas verde azules asimilan amonio del agua intersticial y

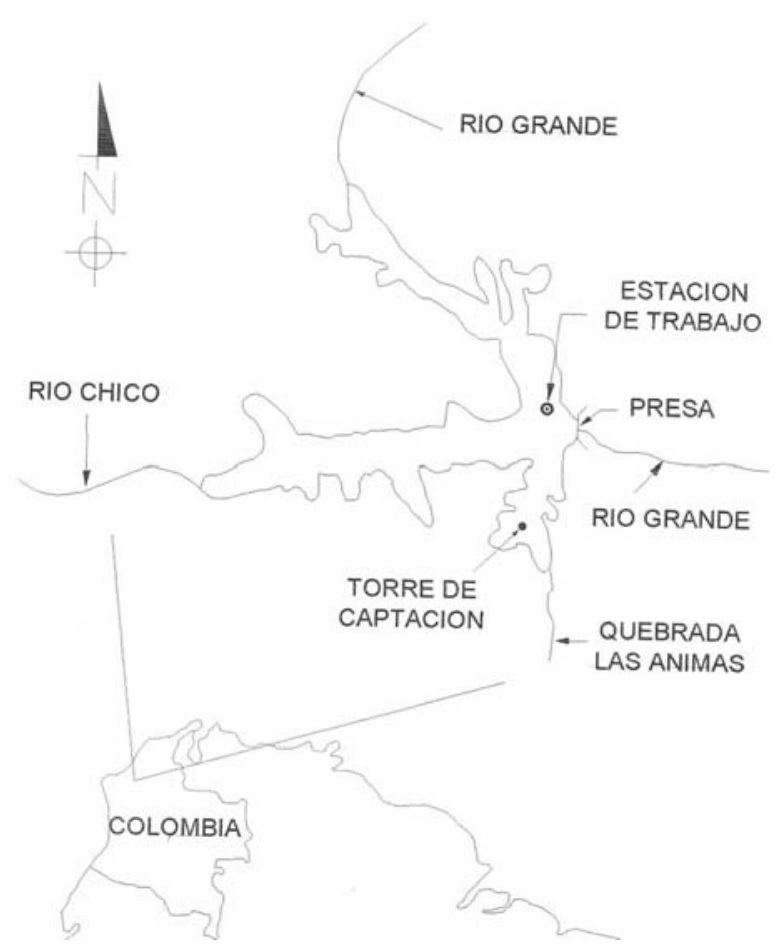

Figura 1. Ubicación del embalse de Río Grande II. Location of the Rio Grande II reservoir.

excretan grandes cantidades de nitrógeno orgánico disuelto a las capas superiores del agua, el cual afecta considerablemente la química del cuerpo de agua.

Las variables mencionadas que afectan los flujos de mineralización han sido evaluadas en zonas templadas, en Colombia este es el primer trabajo publicado sobre mineralización. $\mathrm{Su}$ propósito principal es el de determinar la intensidad de la mineralización y las variables que la condicionan en el eje gravedad-luz en la presa del embalse Río Grande II. Los objetivos de este estudio son: i) Establecer las velocidades de la mineralización y la sedimentación del material partículado en las proximidades de la presa del embalse de Río Grande, ii) Medir los cambios temporales en dichas velocidades y en función de la profundidad de la capa de mezcla, iii) Conocer el grado de mineralización del material partículado hasta llegar a la superficie del sedimento, y iv) Determinar la profundidad a la que hay una mayor tasa de mineralización $\mathrm{y}$ observar si hay cambios temporales en ella. 
Como hipótesis de partida se considera que la profundidad de la altura de mezcla está relacionada con la variación en la concentración del material mineralizado en el eje gravedad-luz. Según dicha hipótesis, en el periodo muestreado, con el aumento de la temperatura hay una mayor mineralización, pero la profundidad a la que ocurre la mayor descomposición está más próxima a la superficie.

\section{MATERIAL Y MÉTODOS}

Los experimentos fueron realizados a cada diez días entre marzo y julio de 2003 cerca de la presa del embalse de Río Grande II $\left(6^{\circ} 32^{\prime} 62^{\prime \prime} \mathrm{N}\right.$, $75^{\circ} 27^{\prime} 27^{\prime \prime} \mathrm{W}$ ), ubicado en el departamento de Antioquia, Colombia (Fig. 1). El embalse se encuentra a una altura promedia de $2270 \mathrm{~m}$, presenta un área superficial de 1200 ha, un volumen de $220 \mathrm{hm}^{3}$, una profundidad máxima de $42 \mathrm{~m}$, una profundidad promedio de $37.9 \mathrm{~m} \mathrm{y}$ una longitud máxima de $10 \mathrm{~km}$.

La temperatura y el oxígeno disuelto se midieron cada $25 \mathrm{~cm}$ hasta los $10 \mathrm{~m}$, de ahí en adelante se midieron a cada metro. La profundidad de la zona de mezcla se evaluó de acuerdo con lo establecido en Reynolds (1984). Adicionalmente, se determinó la profundidad de la zona fótica mediante el uso de un cuantómetro LiCor Li-250.

Con la finalidad de realizar una caracterización general de la columna de agua en la zona de trabajo $(\mathrm{Z}$ media $=35 \mathrm{~m}$ ), se extrajeron muestras de agua cada $5 \mathrm{~m}$ desde la superficie al fondo de la columna; en estas muestras se evaluaron la conductividad $\left(\mu \mathrm{S} \mathrm{cm}^{-1}\right)$, el $\mathrm{pH}$, la alcalinidad $\left(\mathrm{mmol} \mathrm{l}^{-1}\right.$ ), la turbiedad (NTU), $\mathrm{N}-\mathrm{NH}_{4}^{+}, \mathrm{N}-\mathrm{NO}_{3}$ y $\mathrm{P}_{-} \mathrm{PO}_{4}$, todos en $\mathrm{mg} \mathrm{l}^{-1}$.

Para el estudio de la mineralización se utilizaron trampas de PVC con una relación altura/diámetro no mayor de ocho (Bloesch \& Burns, 1980). Al inicio de las labores de muestreo se colocaron dos series paralelas de cinco trampas a una distancia de $10 \mathrm{~m}$ entre ellas; cada una fue llenada previamente con agua destilada y fueron sumergidas lentamente con el fin de disminuir los intercambios con el agua circundante. No se agregó ningún tipo de preservante al agua de la trampa. La primera trampa fue colocada debajo de la zona fótica y la última a cinco metros del fondo; las demás a cada cinco metros entre ellas como se observa en la figura 2. Las trampas permanecieron aproximadamente siete horas dentro del agua (entre las 9:00 y las 16:00 horas).

Al momento de retirar las trampas, los primeros $15 \mathrm{~cm}$ del material colectado fueron vertidos al embalse asumiendo que correspondían al intercambio de agua entre la trampa y la columna de agua; en el volumen de agua restante se midieron inmediatamente el oxígeno disuelto, el porcentaje de saturación y la temperatura con un oxímetro YSI 52. El contenido de cada trampa fue envasado y refrigerado para su transporte. En

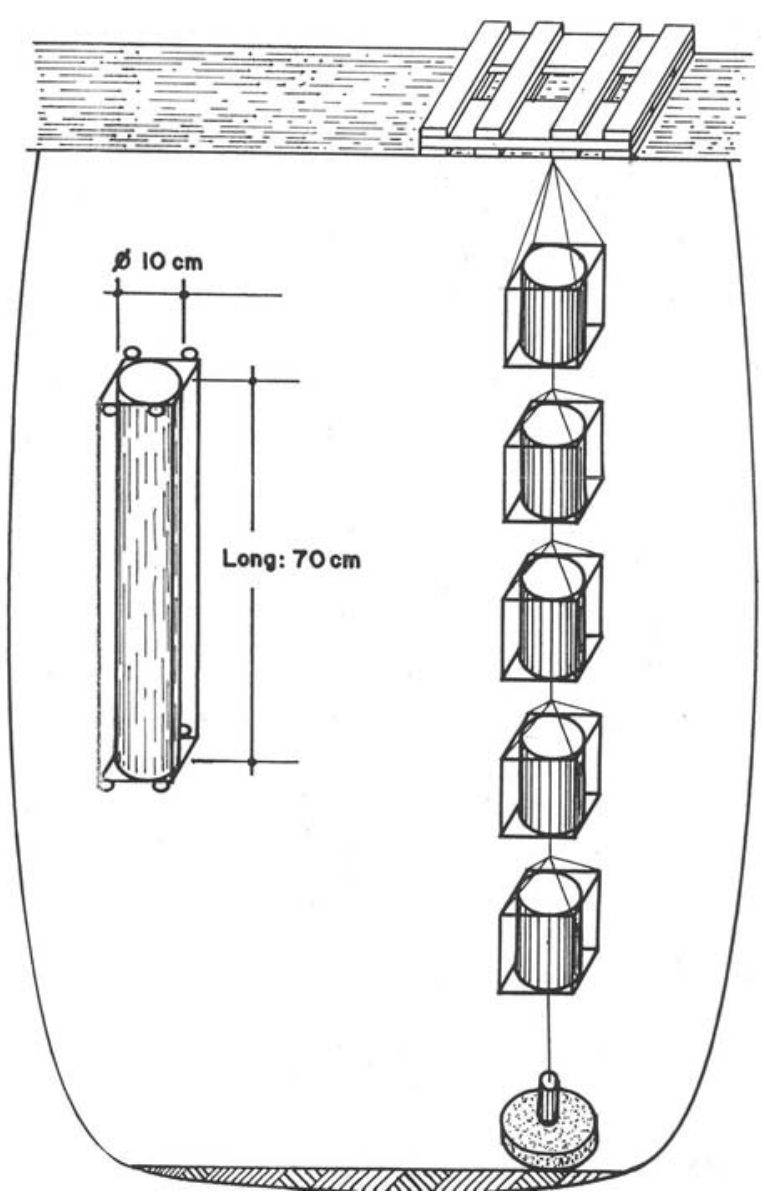

Figura 2. Sistema de trampas empleado en la investigación. Trap system used in this research. 
el laboratorio se cuantificaron las concentraciones de las sales disueltas totales SDT ( $\mathrm{mg} \mathrm{l}^{-1}$ ), sales disueltas fijas SDF $\left(\mathrm{mg} \mathrm{l}^{-1}\right)$ y sales disueltas volátiles SDV $\left(\mathrm{mg} \mathrm{l}^{-1}\right)$. Como evaluadores del material mineralizado a nivel global, se midieron (en muestras de agua previamente filtradas) el nitrógeno amoniacal (mg $\left.\mathrm{l}^{-1} \mathrm{~N}-\mathrm{NH}_{4}^{+}\right)$, los nitratos $\left(\mathrm{mg} \mathrm{l}^{-1} \mathrm{~N}-\mathrm{NO}_{3}\right)$, el fósforo soluble reactivo $\left(\mathrm{mg} \mathrm{l}^{-1} \mathrm{P}^{-} \mathrm{PO}_{4}\right)$, la alcalinidad $\left(\mathrm{mmol} \mathrm{l}^{-1}\right)$ y la demanda química de oxígeno $\left(\mathrm{mg} \mathrm{l}^{-1}\right)$. También se midieron la con- ductividad eléctrica, la turbidez, el $\mathrm{pH}$ y el carbono inorgánico disuelto (CID); este último como la suma de carbonatos, bicarbonatos y $\mathrm{CO}_{2}$ (Mackereth et al., 1972), y la turbidez.

El flujo de mineralización de amonio, nitratos y fosfatos se estimó graficando el logaritmo natural de la concentración liberada de cada nutriente en función de la profundidad a la que fueron colocadas las trampas; la pendiente de la línea representó el flujo de liberación (K). (modificado de Rydin, 2000). A los datos obtenidos

Tabla 1. Resultados del análisis exploratorio de la información colectada en el experimento de mineralización $(n=130)$. Results of the exploratory analysis of the information collected in the mineralization experiment $(\mathrm{n}=130)$.

\begin{tabular}{|c|c|c|c|c|c|}
\hline Variable (unidades) & Factor & Media & Mínimo & Máximo & C.V $(\%)$ \\
\hline \multirow[t]{2}{*}{ Oxigeno (mg/l) } & Profundidad & 2.05 & 0.3 & 6.78 & 68.5 \\
\hline & Muestreo & 2.03 & 0.86 & 4.6 & 40.7 \\
\hline \multirow[t]{2}{*}{ Temperatura $\left({ }^{\circ} \mathrm{C}\right)$} & Profundidad & 18.4 & 17.1 & 21.0 & 4.5 \\
\hline & Muestreo & 18.4 & 17.5 & 19.7 & 3.3 \\
\hline \multirow[t]{2}{*}{ Conductividad $\left(\mu \mathrm{S} \mathrm{l}^{-1}\right)$} & Profundidad & 25.18 & 13.8 & 28.3 & 17.5 \\
\hline & Muestreo & 23.1 & 14.2 & 26.5 & 14.5 \\
\hline \multirow[t]{2}{*}{ pH (unidades de $\mathrm{pH}$ ) } & Profundidad & 7.07 & 6.4 & 10.13 & 9.5 \\
\hline & Muestreo & 7.05 & 6.6 & 9.88 & 8.1 \\
\hline \multirow[t]{2}{*}{ Turbiedad (NTU) } & Profundidad & 35.7 & 0.0 & 956.0 & 253.3 \\
\hline & Muestreo & 34,5 & 3,8 & 945.7 & 87 \\
\hline \multirow[t]{2}{*}{ Fósforo $\left(\mathrm{mg} \mathrm{PO}_{4} \mathrm{l}^{-1}\right)$} & Profundidad & 0.54 & 0.04 & 5.72 & 191.3 \\
\hline & Muestreo & 0.37 & 0.14 & 1.6 & 83.5 \\
\hline \multirow[t]{2}{*}{ N. Amoniacal $\left(\mathrm{mg} \mathrm{NH}_{4}^{+} \mathrm{l}^{-1}\right)$} & Profundidad & 0.34 & 0.01 & 2.47 & 89.7 \\
\hline & Muestreo & 0.34 & 0.14 & 0.7 & 42.3 \\
\hline \multirow[t]{2}{*}{ Nitratos $\left(\mathrm{mg} \mathrm{NO}_{3} \mathrm{l}^{-1}\right)$} & Profundidad & 1.83 & 0.3 & 5.9 & 42.9 \\
\hline & Muestreo & 1.83 & 1.26 & 2.88 & 27 \\
\hline \multirow[t]{2}{*}{ DQO $\left(\mathrm{mg} \mathrm{l}^{-1}\right)$} & Profundidad & 244.9 & 5 & 3950 & 180.2 \\
\hline & Muestreo & 232.7 & 19.7 & 769 & 97 \\
\hline \multirow[t]{2}{*}{$\operatorname{STD}\left(\mathrm{mg} \mathrm{l}^{-1}\right)$} & Profundidad & 90.3 & 10.0 & 343.0 & 45.5 \\
\hline & Muestreo & 90 & 13.5 & 289 & 36.6 \\
\hline \multirow[t]{2}{*}{$\operatorname{STDF}\left(\mathrm{mg} \mathrm{l}^{-1}\right)$} & Profundidad & 37.6 & 0.0 & 130.0 & 72.3 \\
\hline & Muestreo & 36.6 & 0.3 & 128.9 & 70 \\
\hline \multirow[t]{2}{*}{$\operatorname{STDV}\left(\mathrm{mg} \mathrm{l}^{-1}\right)$} & Profundidad & 25.75 & 0.0 & 280 & 66.7 \\
\hline & Muestreo & 25 & 0.25 & 285.7 & 54.8 \\
\hline \multirow[t]{2}{*}{ Alcalinidad $\left(\mathrm{mg} \mathrm{l}^{-1}\right)$} & Profundidad & 0.1898 & 0.048 & 0.52 & 38.3 \\
\hline & Muestreo & 0.18 & 0.087 & 0.5 & 37.6 \\
\hline \multirow[t]{2}{*}{$\mathrm{CO}_{2}$ libre $\left(\mathrm{mmol} \mathrm{l}^{-1}\right)$} & Profundidad & 0.0619 & 0.00001 & 0.62 & 106.1 \\
\hline & Muestreo & 0.0601 & 0,00012 & 0.6 & 104.8 \\
\hline \multirow[t]{2}{*}{$\mathrm{HCO}_{3}\left(\mathrm{mmol} \mathrm{l}^{-1}\right)$} & Profundidad & 0.19 & 0.048 & 0.52 & 38.3 \\
\hline & Muestreo & 0.18 & 0.07 & 0.49 & 37.9 \\
\hline \multirow[t]{2}{*}{$\mathrm{CO}_{3}^{-}\left(\mathrm{mmol} \mathrm{l}^{-1}\right)$} & Profundidad & 0.00144 & 0.00001 & 0.097 & 623.9 \\
\hline & Muestreo & 0.0045 & 0,00004 & 0.095 & 487.4 \\
\hline Altura zona de mezcla (m) & Muestreo & 0.85 & 0.25 & 3.0 & 99.1 \\
\hline Flujo de mineralización de amonio $\left(\mathrm{mg} \mathrm{l}^{-1} \mathrm{~m}^{-1}\right)$ & Muestreo & 0,174 & -0.0649 & 0.9665 & 94.3 \\
\hline Flujo de mineralización de fósforo $\left(\mathrm{mg} \mathrm{l}^{-1} \mathrm{~m}^{-1}\right)$ & Muestreo & $-0,074$ & -10.062 & 0,3374 & -209 \\
\hline Flujo de mineralización de nitrato $\left(\mathrm{mg} \mathrm{l}^{-1} \mathrm{~m}^{-1}\right)$ & Muestreo & 0.00505 & -0.288 & 0.28 & 2239 \\
\hline
\end{tabular}


se les calculó la media aritmética, la desviación estándar y el coeficiente de variación (CV).

Para establecer la existencia de diferencias significativas de los flujos de mineralización entre tiempos de muestreo (13) y profundidades (5), se aplicó un análisis de varianza (ANDEVA) de dos vías con medidas repetidas. La asociación entre los flujos de mineralización y las variables físicas y químicas se efectuó mediante un análisis de regresión y correlación lineal múltiples. Los análisis estadísticos mencionados anteriormente se llevaron a cabo con el paquete estadístico Statgraphics v.5.0.

\section{RESULTADOS}

Variables como temperatura, conductividad y $\mathrm{pH}$ mostraron valores bajos del CV; las demás presentaron valores altos para esta misma variable. Los resultados del ANDEVA mostraron que, con excepción de la turbiedad ( $\alpha=0.1786)$, todas las variables evaluadas presentaron diferencias temporales significativas. La turbiedad, además, presentó la mayor variación durante la investigación (Tabla 1). La variación entre profundidades fue mayor que entre muestreos. Además de la turbiedad (Figs. 4A y 4B), las variables que no presentaron diferencias significativas para este factor fueron las SDT $(\alpha=0.176)$, las $\operatorname{SDF}(\alpha=$ $0.4293)$ y las $\operatorname{SDV}(\alpha=0.7352)$.
La columna de agua en la zona de trabajo presentó un valor medio de $\mathrm{pH}$ muy cercano al neutro (6.58 unidades), acompañado de una baja alcalinidad (18.2 mg $\left.\mathrm{CaCO}_{3} 1^{-1}\right)$ y de unos valores bajos de conductividad $(42.1 \mu \mathrm{S}$ $\mathrm{cm}^{-1}$ ) y turbiedad (17.2 NTU). La figura 3 muestra la variación de la temperatura y del oxígeno disuelto a lo largo de la columna de agua en la zona de la presa del embalse. En ella se puede observar que estas variables presentan perfiles clinogrados, que en algunos muestreos cambiaron a heterógradas, mostrando un máximo de oxígeno metalimnético, el cual es un indicio de un sistema establemente estratificado. Galvez et al. (1989) afirmaron que durante la estratificación la mineralización máxima se alcanza sobre la termoclina cuando el hipolimnion es anóxico. Además, la temperatura en la columna de agua se mantuvo por encima de los $16.2{ }^{\circ} \mathrm{C}$ y en las trampas fue superior a los $17.1{ }^{\circ} \mathrm{C}$ (Figs. 4C y 4D), temperaturas éstas que permiten que la descomposición de la materia orgánica continúe a una tasa considerable, además de la presencia de oxígeno en todas las trampas (Figs. 4E y 4F).

La altura de mezcla osciló entre los 0.25 $\mathrm{m}$ y los $3.00 \mathrm{~m}$ lo que indicaría que el sistema permaneció estratificado con una mezcla superficial muy leve (Tabla 2). En esos primeros metros se observa que la concentración de oxígeno fue alta y la temperatura uniforme.

Tabla 2. Capa de mezcla y flujos de mineralización de amonio, fósforo y nitratos en las dos réplicas durante el experimento $(p=$ pendiente, $R=$ replica). Mixing depth and mineralization fluxes of ammonium, phosphorus, and nitrates in the two replicates during the experiment $(\mathrm{p}=$ slope, $\mathrm{R}=$ reply).

\begin{tabular}{crrrrrrr}
\hline Fecha & Altura de mezcla $(\mathrm{m})$ & $p$ Amonio $R 1$ & $p$ Amonio $R 2$ & $p$ Fósforo $R 1$ & $p$ Fósforo $R 2$ & $p$ nitratos $R 1$ & $p$ nitratos $R 2$ \\
\hline $28 / 03 / 2003$ & 0.75 & 0.7447 & 0.9665 & -0.076 & -0.112 & -0.0634 & 0.1317 \\
$07 / 04 / 2003$ & 0.25 & 0.0441 & 0.266 & 0.1694 & -0.2541 & 0.28 \\
$16 / 04 / 2003$ & 0.5 & 0.3744 & 0.227 & -0.148 & 0.2148 & 0.0749 & 0.0226 \\
$28 / 04 / 2003$ & 0.5 & -0.0649 & 0.5006 & 0.3374 & 0.014 & 0.035 & 0.102 \\
$12 / 05 / 2003$ & 0.75 & 0.397 & 0.252 & -0.213 & -0.8957 & -0.051 & -0.2883 \\
$20 / 05 / 2003$ & 1 & 0.129 & 0.174 & -0.066 & -0.5029 & 0.14 \\
$29 / 05 / 2003$ & 0.25 & 0.4302 & 0.9215 & -0.5751 & -0.072 & 0.0319 & 0.1823 \\
$09 / 06 / 2003$ & 0.25 & 0.7563 & 0.2145 & -1.000 & -0.5303 & 0.042 & -0.0899 \\
$19 / 06 / 2003$ & 0.75 & 0.0396 & 0.095 & -0.917 & 0.071 & -0.0459 & -0.1707 \\
$01 / 07 / 2003$ & 0.5 & 0.2594 & 0.2042 & -0.1554 & -0.138 & -0.0299 & -0.124 \\
$11 / 07 / 2003$ & 2.25 & 0.123 & 0.069 & 0.196 & -0.0132 & 0.0503 & 0.008 \\
$21 / 07 / 2003$ & 3 & 0.13 & 0.116 & 0.079 & 0.0968 & 0.0644 & 0.0501 \\
\hline
\end{tabular}



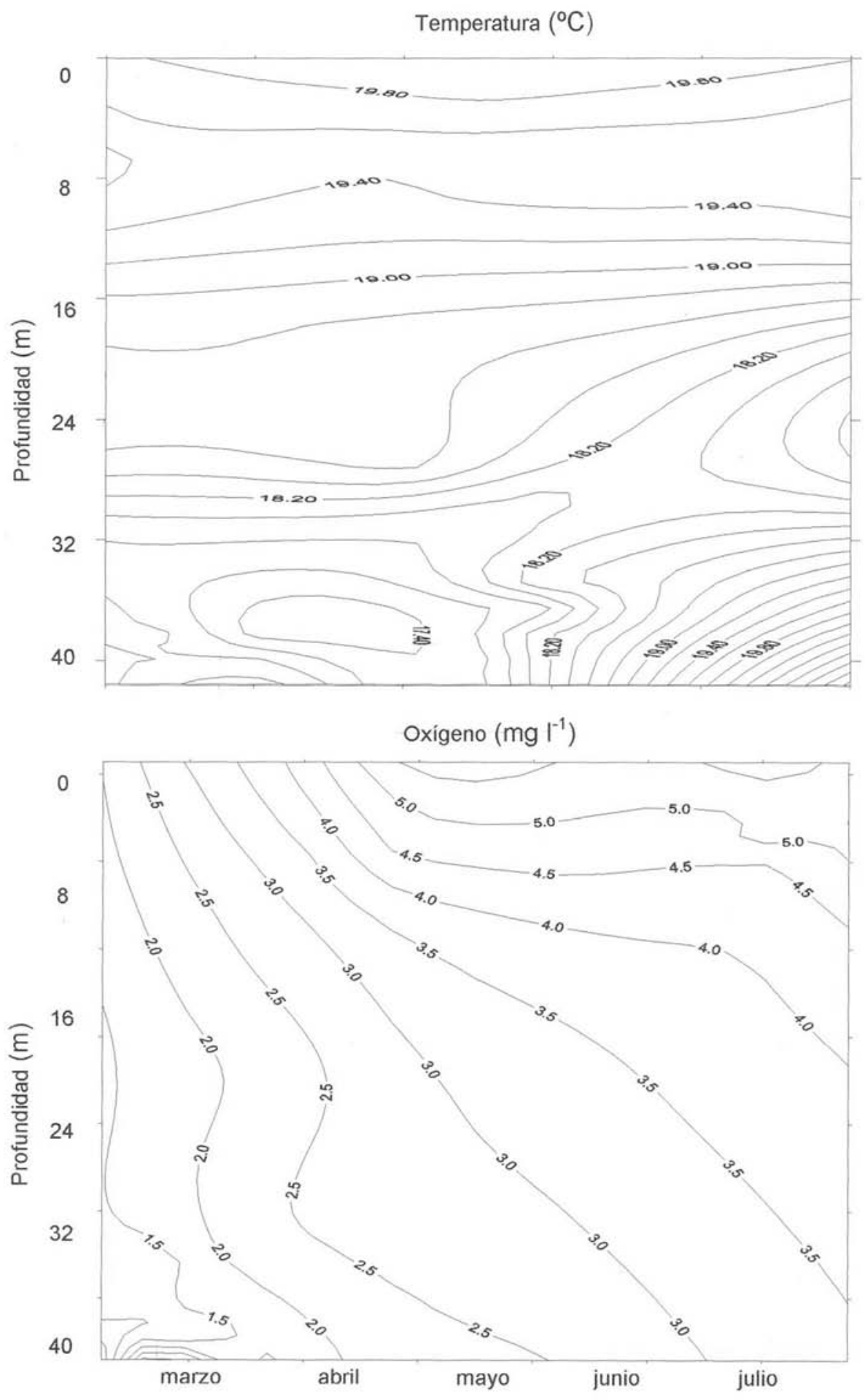

Figura 3. Evolución temporal y espacial de la temperatura y de la concentración de oxígeno disuelto durante el período estudiado en el embalse de Río Grande. Temporal and spatial evolution of temperature and dissolved oxygen concentration during the studied period in the Rio Grande reservoir. 
Tabla 3. Resultados del análisis exploratorio y de varianza de los flujos de mineralización en el tiempo ( $n=13)$. Results of the exploratory and variance analysis of flux mineralization through time.

\begin{tabular}{crrrrrrrr}
\hline Flujo de mineralización & Media & Mínimo & Máximo & Amplitud & CV (\%) & Factor & F & $\alpha$ \\
\hline Amonio & 0.658 & -0.1522 & 2.2264 & 2.3786 & 85.4 & Muestreos & 3.17 & 0.0345 \\
Fósforo & -0.158 & -2.3073 & 0.7741 & 3.0814 & -198.7 & Muestreos & 1.64 & 0.2164 \\
Nitratos & 0.079 & -0.6621 & 6.69 & 7.3521 & 362.8 & Muestreos & 1.8 & 0.1703 \\
\hline
\end{tabular}

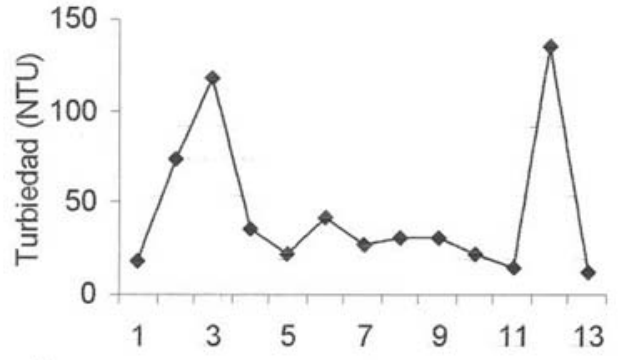

A
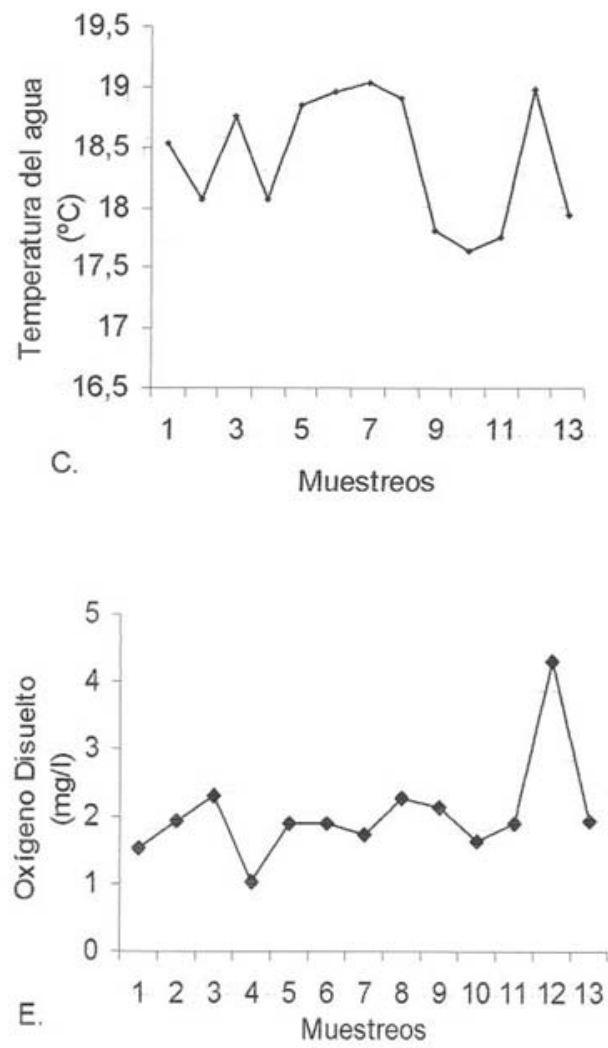
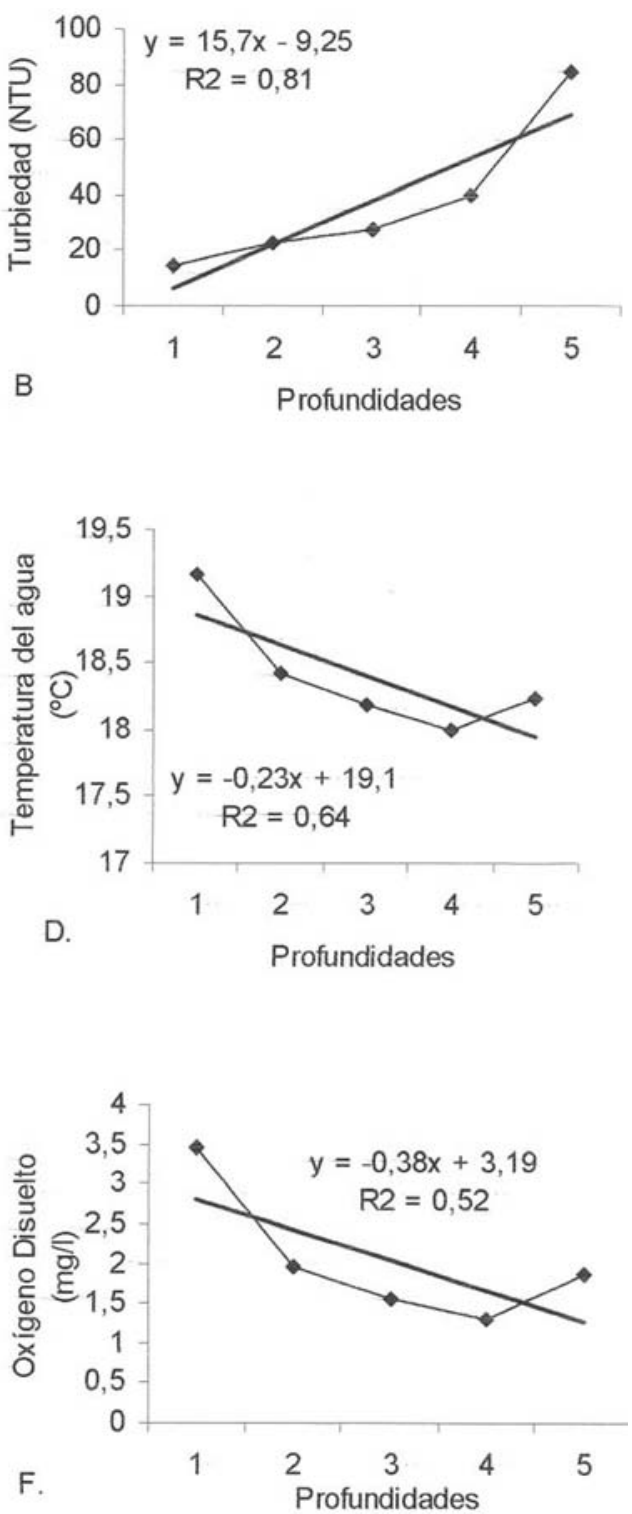

Figura 4. Variación de la turbiedad en el tiempo (A); entre profundidades (B); la temperatura del agua en las trampas en el tiempo (C) y entre las profundidades (D); el oxígeno disuelto en las trampas en el tiempo (E) y entre las profundidades (F). Turbidity variation over time (A); among depths $(B)$; the temperature of the water in the traps over time $(C)$, and among depths $(D)$; the oxygen concentration of the water in the traps over time $(E)$, and among depths $(F)$. 
A partir de los $5 \mathrm{~m}$ aproximadamente la concentración de oxígeno y la temperatura del agua caen de forma abrupta, por lo que se puede afirmar que esta investigación se realizó en condiciones de estratificación.

El análisis de regresión múltiple mostró que el flujo de liberación de amonio estuvo relacionado inversamente con la concentración de oxígeno disuelto $(r=-0.25, \alpha=0.0052)$, el pH $(r=$ -0.38, $\alpha=0.0000)$, con el $\mathrm{CO}_{3}^{-}(r=-0.24, \alpha=$ $0.0086)$ y con la altura de mezcla $(r=-0.21, \alpha=$ $0.0183)$; y directamente con la turbidez $(r=0.62$, $\alpha=0.0000)$, la DQO $(r=0.36, \alpha=0.0000)$ y con la temperatura del agua $(r=0.46, \alpha=0.0176)$.

$\mathrm{Ni}$ los flujos de liberación de los nitratos $(\alpha=$ $0.1703)$ ni el del fósforo soluble $(\alpha=0.2164)$ fueron significativos; sólo el amonio mostró diferencias significativas entre muestreos $(\alpha=0.0345)$. Se presentó una relación significativa entre la temperatura del agua y el flujo de mineralización del amonio, con una liberación de amonio de $0.17 \mathrm{mg}$ de $\mathrm{NH}_{4}^{+} \mathrm{l}^{-1} \mathrm{~m}^{-1}$ en promedio, equivalente a $0.6 \mu \mathrm{g}$ de $\mathrm{NH}_{4}^{+} \mathrm{m}^{-3} \mathrm{~d}^{-1}$ para un promedio de 7 horas de exposición (Figs. 5A y 5B).

Los flujos de liberación del fósforo soluble reactivo no fueron estadísticamente significativos (Tabla 3). Sin embargo se presentaron diferencias significativas entre los periodos de muestreo y entre las profundidades para las concentraciones del fósforo soluble (Figs. 5C y 5D).

La prueba de regresión múltiple mostró que el flujo de liberación de fósforo esta relacionado con la liberación de nitratos $(r=0.23, \alpha=$ $0.0102)$, el $\mathrm{HCO}_{3}^{-}(r=-0.26, \alpha=0.0031)$ y la temperatura del agua $(R=0.27, \alpha=0.0020)$.

La concentración media de nitratos en las trampas fue de $1.83 \mathrm{mg} \mathrm{l}^{-1}$, la cual fue 5 veces mayor que la concentración de amonio con una variación del $89.7 \%$ y con diferencias significativas entre periodos de muestreo ( $\alpha$ $=0.0000)$ pero no entre profundidades $(\alpha$ $=0.5686$ ) (Figs. 5E y 5F). Aunque en la figura anterior se observan dos tendencias, una desde la trampa 1 a la 4 en la que hay un aumento en la concentración de los nitratos con la profundidad. La otra tendencia presentada en la trampa 5 es a la disminución de la concentración con la profundidad, debido a un proceso de desnitrificación muy intenso en el fondo. La prueba de regresión múltiple muestra que el flujo de liberación de nitratos estuvo significativamente relacionado con la temperatura del agua $(r=-0.10, \alpha=0.0254)$, el flujo de liberación de fósforo $(r=-0.24, \alpha=$ $0.0064)$, la alcalinidad ( $r=-0.32, \alpha=0.0003)$, la altura de mezcla $(r=-0.19, \alpha=0.0362)$ y los $\operatorname{SDF}(r=0.33, \alpha=0.0002)$.

\section{DISCUSIÓN}

La temperatura del agua en las trampas presentó baja variación temporal y diferencias significativas entre profundidades, las cuales indican que las trampas más superficiales, localizadas al comienzo de la zona afótica, se diferencian del resto, ubicadas hacia la parte media de la zona afótica y cerca del final de la misma. El fósforo y el nitrógeno amoniacal responden al mismo patrón, siendo las trampas más superficiales diferentes en su comportamiento respecto a las demás. Al evaluar las flujos de mineralización se observan en general diferencias entre sus tendencias las cuales oscilaron entre valores positivos y negativos, aunque la tendencia general de los flujos de mineralización del fósforo y de los nitratos fue una pendiente negativa, lo que significa su disminución con la profundidad, y su dependencia de la temperatura circundante por su cercanía a la termoclina (Fig. 5).

Los flujos de liberación del amonio, el nitrato y el fósforo presentaron una alta variabilidad espacio temporal ya que en cada uno de ellos se obtuvieron valores positivos (liberación) y valores negativos (consumo). Cuando grandes cantidades de materia orgánica sedimentable alcanzan el hipolimnion de un lago estratificado, el amonio se libera y acumula debido a la anoxia hipolimnética. Por ello, el flujo de amonio fue 8.3 veces mayor que el de nitratos, pues el amonio es asimilado en mayor proporción. La concentración de amonio se incrementó con la profundidad y el flujo de mineralización presentó una pendiente positiva, lo que implica que por debajo del metalimnion ocurren las mayores liberaciones de amonio. El material que se sedi- 
menta en un ambiente de aguas cálidas con bajos niveles de oxígeno, incide en la producción de amonio. Wu (2001) encontró que el amonio se incrementa hacia el fondo relacionado con el incremento vertical de su concentración dentro del agua intersticial de los sedimentos y confirma que existe transformación entre varias formas de nitrógeno en este compartimiento ecosistémico.
El mismo autor afirma que la regeneración del nitrógeno ocurre principalmente en la interfase agua-sedimento y el nitrógeno se recicla principalmente en forma de $\mathrm{NH}_{4}^{+}$, aunque también se presenta regeneración de nitrógeno en el epilimnion en menor proporción y principalmente bajo la forma de nitratos. Estudios realizados en varios sistemas leníticos tropicales (Mitamura et
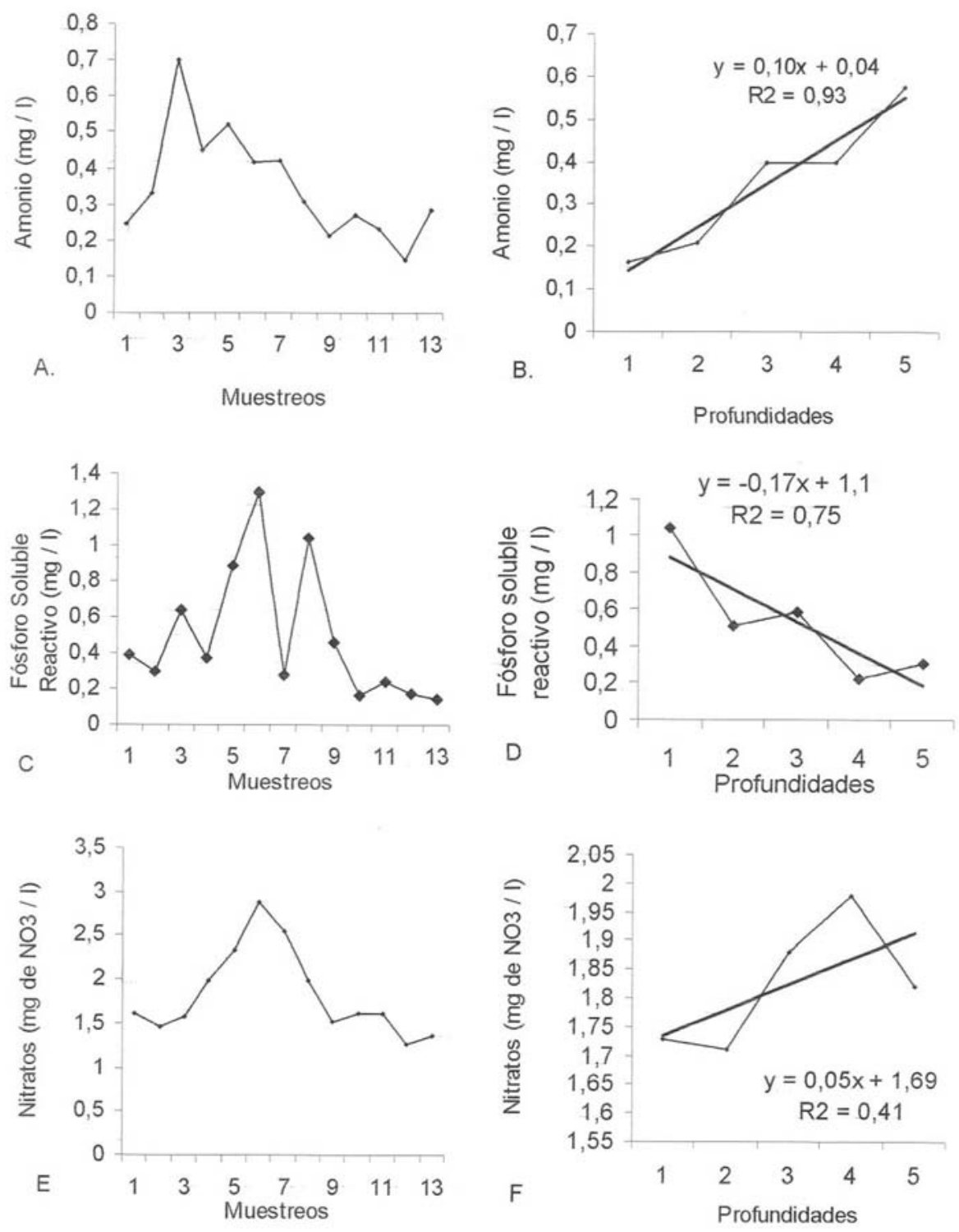

Figura 5. Variación promedio del flujo de: amonio en el tiempo (A) y entre profundidades (B); el FSR en las trampas en el tiempo (C) y entre las profundidades (D); los nitratos en las trampas en el tiempo (E) y entre las profundidades (F). Average variation of the fluxes of: over time $(A)$ and among depths $(B) ;$ the SRP in the traps over time $(C)$, and among depths $(D)$; the nitrates in the traps over time $(E)$ and among depths $(F)$. 
al., 1990; Pinto-Coelho \& Araüjo, 1998; Chalar \& Tundisi, 1999) muestran que el amonio y la urea son utilizados preferiblemente por las comunidades fitoplanctónicas en la capa eufótica y que el flujo de renovación de los nutrientes nitrogenados se incrementa con la profundidad.

Como la temperatura hipolimnética se mantuvo sobre $\operatorname{los} 18^{\circ} \mathrm{C}$, es de esperar que la descomposición del material que se sedimenta y del que se halla en suspensión, continua aún en el fondo del cuerpo de agua. Aunque el oxígeno a partir de los $14 \mathrm{~m}$ fue inferior a $1 \mathrm{mg} \mathrm{l}^{-1}$, la descomposición puede continuar empleando otros aceptores de electrones, como el $\mathrm{NO}_{3}^{-}$, el Fe${ }^{3+}$, el $\mathrm{SO}_{4}^{2-}$ y el $\mathrm{CO}_{2}$ (Ohle, 1956; Håkanson \& Jansson, 2002). Por ello, la relación positiva entre la temperatura del agua y el flujo de mineralización del amonio. Sin embargo, es necesario aclarar que la degradación anaeróbica de los detritos es menos eficiente que la aeróbica (Pérez-Rojas et al., 2000) por lo que la materia orgánica en diferentes estados de descomposición se acumula progresivamente en los sedimentos. Seitzinger (1988) afirma que la desnitrificación ocurre en aguas sólo cuando la concentración de oxígeno es menor que $0.2 \mathrm{mg} \mathrm{l}^{-1}$.

El valor medido de la temperatura de las trampas de fondo fue mayor que el valor medio de las dos trampas superiores (trampas 3 y 4); esta tendencia fue similar a la del oxígeno disuelto y la turbidez, lo que puede ser indicio de que se presentaron corrientes de fondo que contribuyeron a la resuspensión de los sedimentos, a la degradación de la materia orgánica y a generar un proceso de mezcla hipolimnética que puede contribuir con la recirculación de nutrientes esenciales.

La prueba de análisis de varianza para el amonio mostró que las trampas más superficiales ( $\left(\begin{array}{lll}1 & \text { y }\end{array}\right)$ se diferenciaron notoriamente del resto de trampas que conformaban el sistema (trampas 3, 4 y 5). En las primeras, la concentración de amonio fue menor, quizás por el consumo del fitoplancton y el bacterioplancton y por la nitrificación, procesos estos que disminuyen su incidencia con el aumento en la profundidad y la disminución del oxígeno disuelto. Por ello, la concentración del mismo y su flujo de mineralización aumentaron hacia el hipolimnion. Vale la pena anotar que se presentó una velocidad media del viento de $4.69 \mathrm{~m} \mathrm{~s}^{-1}$ la cual puede generar corrientes de circulación de Langmuir (Reynolds, 1984), lo que podría ocasionar sobre-atrapamiento de material sestónico en las trampas afectadas.

La demanda química de oxígeno presentó una relación significativa con el amonio, asociada con el aumento de la DQO con la profundidad, indicativo de que el material demandante de oxígeno puede tener atrapadas grandes cantidades de nitrógeno las cuales se liberan lentamente en forma de amonio. El aumento en la capa de mezcla acompañado de los vientos continuos que se generaron en la zona de la presa, producen un epilimnion bien mezclado y oxigenado, que da lugar a la nitrificación del amonio y a su consumo, como ya se mencionó. A nivel de las trampas podría esperarse que al aumentar la zona de mezcla se genere una disminución en los flujos de amonio.

El flujo de mineralización del fósforo presentó en general una tendencia inversa al flujo de mineralización del amonio, es decir, disminución con la profundidad. En los lagos tropicales, debido a su alta temperatura, el metabolismo de los organismos aumenta considerablemente, ocasionando que los ortofosfato sean más rápidamente asimilados e incorporados como biomasa. Este es uno de los principales motivos por los que en estos lagos, exceptuando los artificialmente eutrofizados, la concentración de ortofosfato es muy baja (Esteves, 1998). Esta afirmación no se ajusta a los datos encontrados en la presente investigación, ya que se halló que en las trampas más superficiales la concentración de fósforo era mayor. A menos, que se considere que estos valores responden a otro tipo de aportes o a que en la superficie debido a su elevada temperatura, la liberación sea mayor $(r=0.28, \alpha=0.0020)$ y su consumo es restringido por la limitación debida a los bajos niveles de amonio en la superficie y/o por fotoinhibición. Los trabajos de Ohle (1937) y Einsele (1938) citados en Esteves (1998) muestran la existencia de fuertes alteraciones del patrón general de ciclaje de nutrientes. Ohle (1958), citado por el mismo autor, demostró que la liberación de la mayor parte 
del fósforo desde los detritos ocurría en el epilimnion, mucho antes de ser sedimentado.

El flujo de liberación promedia de fósforo fue de $-0,074 \mathrm{mg}$ de $\mathrm{PO}_{4} \mathrm{l}^{-1} \mathrm{~m}^{-1}$. Este valor implica que es un flujo de pérdida del mismo; hecho este que no es muy común en el trópico pues el patrón general de distribución del fósforo muestra una curva clinograda inversa, es decir, con tendencia a aumentar con la profundidad. Como en los demás ciclos biogeoquímicos, en el ciclo del fósforo las bacterias juegan un papel muy importante, pues son las responsables de la descomposición de la materia orgánica. En este proceso ocurre liberación de fosfato hacia el medio en forma inorgánica en ambientas anóxicos (Esteves, 1998).

En la superficie según se había discutido anteriormente, la concentración de fósforo fue anormalmente alta y hacia el fondo de la zona de la presa la concentración de este nutriente tendió a disminuir, lo que refleja el flujo de pérdida mencionado en el eje gravedad-luz. Los factores de pérdida citados más comúnmente son: el consumo por los microorganismos, las pérdidas por lavado hidráulico, la adsorción de fosfato por arcillas y metales como el calcio y el hierro y la sedimentación en la que se pierde la fracción orgánica e inorgánica partículada del fósforo. La resuspensión de partículas del sedimento puede facilitar un incremento en el atrapamiento del fósforo por parte de las partículas presentes en la columna de agua. Se sabe que las arcillas compiten con el plancton por iones como el fosfato y bajo condiciones apropiadas pueden transportar el fósforo fuera de la columna de agua a través de la sedimentación (Lind et al., 1999).

Adicionalmente, las corrientes de densidad también pueden influenciar la distribución de los nutrientes en los embalses estratificados. Durante los periodos de estratificación térmica, las diferencias verticales en la densidad y las diferencias entre la densidad de los afluentes y los estratos de agua del reservorio controlan la posición vertical de ingreso de los tributarios y los nutrientes que estos transportan (Thornton et al., 1990). La redistribución del fósforo inorgánico liberado es controlada por la capacidad adsortiva del fosfato, dependiente del $\mathrm{pH}$ en los sedimentos, y por el flujo de mineralización y adsorción. Cabe anotar que el $\mathrm{pH}$ decrece por la producción de gas carbónico durante el proceso de descomposición (Istvánovics, 1988).

El descenso en la concentración de nitratos hacia el fondo es debido posiblemente a un proceso de desnitrificación muy intenso en el fondo. Wetzel (2001) plantea que la remoción del nitrógeno es usualmente dominada por la desnitrificación, la cual es directamente correlacionada con la carga de materia orgánica. Incluso en los embalses eutrofizados, establemente estratificados y con hipolimnion anóxico, como el estudiado, el nitrógeno puede salir del sistema en forma gaseosa (Margalef, 1983). La nitrificación es un proceso que permite la oxidación del amonio para producir nitratos y nitritos, pero esta requiere de oxígeno, por lo que en las primeras dos trampas se encontraron valores parecidos a la concentración de este nutriente. La asimilación del nitrato es normalmente menor que la asimilación relativa del amonio, aunque las concentraciones de nitrato y amonio presenten magnitudes similares (Axler et al., 1981).

La disminución de la tasa de degradación de los nitritos y nitratos en ambientes con altas concentraciones puede ser atribuida a la desnitrificación de los nitratos en nitritos (Awadellah et al., 1998). Muchas bacterias en condiciones anaeróbicas son capaces de utilizar el nitrato como aceptor de hidrógeno, reduciéndolo a nitrógeno molecular o amonio. Otras bacterias lo pueden utilizar como aceptor de electrones en la cadena respiratoria (Esteves, 1998). El hipolimnion casi anóxico de un embalse tropical facilita la ocurrencia de los fenómenos anteriormente mencionados ya que su mayor temperatura respecto a los de zona templada, implica una mayor dinámica en los procesos metabólicos de los microorganismos. El mismo autor afirma que la respiración del nitrato, corresponde bioquímicamente a la respiración del oxígeno y el sustrato oxidado a gas carbónico es el agua, con una ganancia de energía inferior al $10 \%$ de la respiración aeróbica. Esta producción de $\mathrm{CO}_{2}$ libre genera patrones de variación de la alcalinidad y del CID. 
La concentración máxima de nitratos probablemente es debida a la regeneración oxidativa de nutrientes (Wu et al., 2001). El aumento de la capa de mezcla reduce la capa hipolimnética lo que se refleja en una disminución de la desnitrificación, que afecta la concentración de nitrato en el medio acuático.

\section{CONCLUSIONES}

El flujo de mineralización del fósforo presenta un valor negativo durante la investigación, lo que se asoció a una tasa de consumo y/o atrapamiento permanente en el sistema, las cuales pueden estar gobernadas por la hidrodinámica del embalse. Esto no implicaría al fósforo como el nutriente limitante de la productividad en el embalse. Además, el fósforo mostró una tendencia a disminuir su concentración con la profundidad, un patrón poco común para un embalse tropical estratificado. El flujo de mineralización de los nitratos presentó un valor positivo muy pequeño que no varió significativamente entre muestreos, por lo que el consumo supera la liberación con el aumento en la temperatura del agua y de la altura de mezcla. El flujo de mineralización del amonio es sensible a la altura de la capa de mezcla y a la variación en la temperatura del agua, mostrando una mayor mineralización en los primeros muestreos en los cuales la temperatura fue mayor. Con el aumento en la altura de la capa de mezcla se evidenció que disminuye la mineralización del amonio y la profundidad a la que ocurre no varía, manteniéndose la mayor descomposición de la materia orgánica sobre la segunda trampa. Los patrones de circulación en el embalse permiten que se presenten corrientes hipolimnéticas, las cuales atrapan fósforo y lo sacan del sistema conduciéndolo hacia la torre de captación, ya que este sistema se caracteriza por presentar una fuerte asimetría en el flujo debido a que las dos colas principales se unen en los alrededores de la presa, para luego verter sus aguas en la zona de la torre de captación aguas abajo.

\section{AGRADECIMIENTOS}

A los profesores Gustavo Peñuela y Gabriel Guillot por lectura del manuscrito y sugerencias, a dos evaluadores inéditos por sus comentarios y sugerencias, a Boris Montoya por la elaboración de las figuras y a las Empresa Públicas de Medellín por facilitar sus instalaciones para el trabajo de campo.

\section{BIBLIOGRAFÍA}

ALVAREZ COBELAS, M., D. J. VELASCO, \& A. RUBIO OLMO. 1993. Settling seston in a hypertrophic lake. Arch. Hydrobiol, 127 (3): 327343.

AMON, M. W. R. \& R. BENNER. 1996. Bacterial utilization of different size classes of disolved organic matter. Limnol. Oceanogr., 41 (1): 41-51.

APHA, AWWA \& WPCF. 1995. Standard methods for the examination of water and wastewaters. $18^{\mathrm{a}}$ ed. United Book Press, Inc., Baltimore. 1268 pp.

AWADALLAH, R. M., M. S. SLTAN \& M. N. SHABER. 1998. Bacterial removal of nitrate, nitrite and sulphate in wastewater. Wat. Res, 32 (10): 3080-3084.

AXLER, R. P., G. W. REDFIELD \& C. R. GOLDMAN. 1981.The importance of regenerated nitrogen to phytoplankton productivity in a subalpine lake. Ecology, 62 (2): 345-354.

BLOESCH, J. \& M. BURNS. 1980. A critical review on sediment traps technique. Schweiz. Z. Hydrobiol, 42 (1): 15-55.

BOERS, P. \& V. HESE. 1988. Phosphorus release from the peaty sediments of the Loodsdrecht lakes (The Netherlands). Wat. Res, 22 (3): 355-363.

CHALAR, G. \& TUNDISI, J. 1999. Main processes in the water column determined by wind and rainfall at lobo (broa) reservoir. Implications for phosphorus cycling. In: Theoretical reservoir ecology and it's applications. J. G. Tundisi \& M. Straskraba (eds.): 53-65. International Institute of Ecology, Sao Carlos.

COLE, J. \& G. LIKENS. 1979. Measurements of mineralization of phytoplankton detritus in an oligotrofic lake. Limnol. Oceangr., 24 (3): 541547.

ESTEVES, F. 1998. Fundamentos deLimnología. Interciencia. Rio de Janeiro. $2^{\mathrm{a}}$ ed. 602 pp. 
GALVEZ, J. A., F. X. NIELL, \& J. LUCENA. 1989. Seston vertical flux model for an eutrophic reservoir. Arch. Hydrobiol. Beih. Ergebn. Limnol, 33: 9-18.

GOMEZ, E., C. DURILLON, G. ROFES, \& B. PICOT. 1999. Phosphate adsorption and release from sediments of Brack lagoons: $\mathrm{pH}, \mathrm{O}_{2}$ and loading influence. Wat. Res., 33 (10): 2437-2447.

HÅKANSON, L. \& M. JANSSON. 2002. Principles of lake sedimentology. Blackburn Press. New Jersey. $316 \mathrm{pp}$.

HOWARTH, W. R., R. MARINO, \& J. COLE. 1988. Nitrogen fixation in freshwater, estuarine, and marine ecosystems. 1. Biogeochemical controls. Limnol. Oceangr., 33: 688-701.

ISTVÁNOVICS, V. 1988. Seasonal variation of phosphorus release from the sediments of shallow lake Balaton (Hungary). Wat. Res., 22: 1473-1481.

LEHMAN, J. T. 1980. Release and cycling pf nutrients between planktonic algae and herbivores. Limnol. Oceangr., 25: 620-632.

LIND, O. T. \& L. DAVALOS-LIND. 1999. Suspended clay: it's role in reservoir productivity. In: Theoretical reservoir ecology and it's aplications. J. G. Tundisi \& M. Straskraba (eds.): 85-97. International Institute of Ecology, Sao Carlos.

MACKERETH S. J., J. HERON, J. F. TALLING. 1978. Water analysis; some revised methods for limnologist. Freshwater Biological Association. Scientific Publications No 36. Titus Wilson \& Sons Ltda. Kendall. 120 pp.

MARGALEF, R. 1983. Limnología. Ed. Omega. Barcelona. $1010 \mathrm{pp}$.

MITAMURA, O., SAIJO, Y., HINO, K. \& BARBOSA, R. F. A. 1990. The significance do regenerated nitrogen for phytoplankton productivity in lakes Dom Helvécio, Jacaré and Carioca. In: Limnological studies on the Rio Doce Valley Lakes, Brazil. R. L. Bozelli, F. A. Esteves \& F. Roland (eds.): 129-153. Ed. UFRJ/SBL. Rio de Janeiro.

OHLE, W. 1956. Bioactivity, production, and energy utilization of lakes. Limnol. Oceangr., 1: 139-149.

PERKINS, R. G. \& G. J. UNDERWOOD. 2001. The potencial for phosphorus release across the sediment-water interface in a eutrophic reservoir dosed with ferric sulphate. Wat. Res., 35: 1399-1406.
PÉREZ-ROJAS, A., B. TORRES-OROZCO, E. MORALEZ-GUTIERREZ, \& E. PÉREZ-MÉNDEZ. 2000. Textura, composición y contenido de materia orgánica de los sedimentos recientes de un lago tropical de México. Hidrobiológica, 10: 41-50.

PINTO-COELHO, R. M. \& ARAÜJO, M. A. 1998. Efeitos da remoçao de macrófitas sobre disponibildae de nitrato e amonia e no zooplancton de um reservatório eutrófico raso. Anais Do VIII Seminario Regional de Ecología, 1217-1223. UFSCar, Sao Carlos-SP.

RATSAK, H., K. MAARSEN, \& A. L. KOOIJMAN. Effects of protozoa on carbon mineralization in activated sludge. Wat. Res., 30: 1-12.

REYNOLDS, C. S. 1984. The ecology of Freshwater Phytoplankton. Cambridge Univ. Press, Cambridge, $384 \mathrm{pp}$.

RYDIN, E. 2000. Potencially mobile phosphorus in lake Erken Sediment. Wat. Res., 34: 2037-2042.

SEITZINGER, P. S. 1988. Denitrification in freshwater and coastal marine ecosystems: Ecological and geochemical significance. Limnol. Oceangr., 33: 702-724.

SERRUYA, C., M. EDELSTEIN, U. POLLINGHER, \& S SERRUYA. 1974. Lake kinneret sediments: Nutrient composition of the pore water and mud mater exchanges. Limnol. Oceangr., 19: 489-508.

THORNTON, K. W., B. L. KIMMEL \& F. E. PAYNE. 1990. Reservoir Limnology: Ecological Perspectives. John Wiley \& Sons, Inc., New York. $246 \mathrm{pp}$.

VADSTEIN, O., L. M. ULSEN, A. BUSCH, T. ANDERSEN \& H. R. REINERTSEN. 2003. Is phosphorus limitation of planktonic heterotrophic bacteria and accumulation of degradable DOC a normal phenomenon in phosphorus-limited systems? A microcosm study. Fems. Micro. Ecol., 46 (3): 3017-316.

WETZEL, R. G. 2001. Limnology: Lake and river ecosystems. $3^{\mathrm{a}}$ ed. Academic Press. San Diego. $1006 \mathrm{pp}$.

WU, F., H. QING \& G. WAN. 2001. Regeneration of $\mathrm{N}, \mathrm{P}$, and $\mathrm{Si}$ near the sediment water interface of lakes from Southwestern China Plateu. Wat. Res., 35 (5): 1334-1337. 
